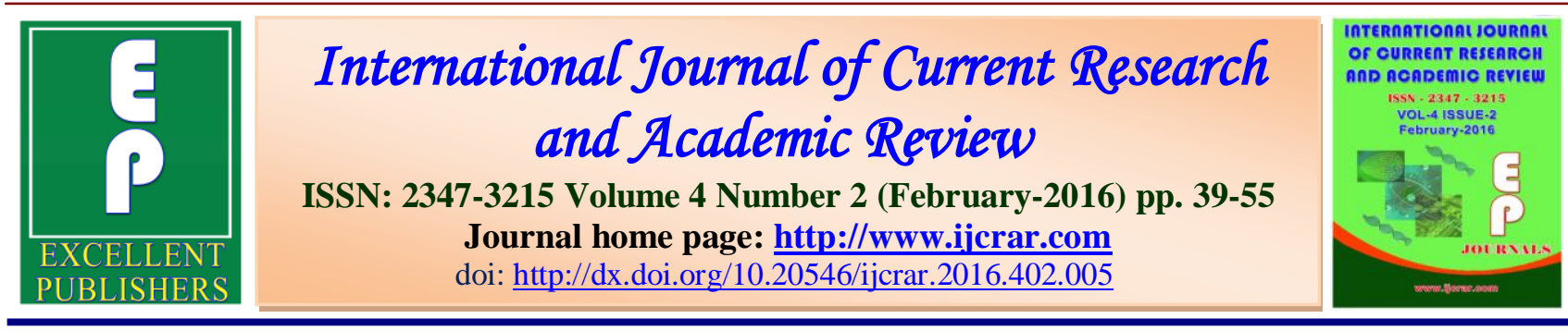

\title{
Studies on Soil Mycoflora in Coastal Area of Tuticorin Dt., Tamilnadu, India
}

\author{
G. Ashok ${ }^{1 *}$, G. Senthilkumar ${ }^{1}$, A. Panneerselvam ${ }^{1}$ and K. Umamagheswari ${ }^{2}$ \\ ${ }^{1}$ Research Department of Botany and Microbiology, A.V.V.M Sri Pushpam College \\ (Autonomous), Poondi - 613 503, Thanjavur, Tamil Nadu, India \\ ${ }^{2}$ Department of Chemistry, Manonmaniam Sundaranar University, Tirunelveli-627 012, India \\ *Corresponding author
}

\begin{tabular}{|c|c|}
\hline KEYWORDS & A B S T R A C $\mathbf{T}$ \\
\hline $\begin{array}{l}\text { Soil Mycoflora, } \\
\text { Coastal Area } \\
\text { Tuticorin, India, } \\
\text { Aspergillus, } \\
\text { Penicillium, } \\
\text { Alternaria, } \\
\text { Curvularia, } \\
\text { Fusarium }\end{array}$ & $\begin{array}{l}\text { A total of } 39 \text { isolates of mycoflora were isolated from soil samples collected } \\
\text { from coastal area of Tuticorin Dt., Tamil Nadu, India. The soil mycoflora } \\
\text { were isolated by using soil dilution technique. Based on morphological } \\
\text { characteristics, fourteen genera of mycoflora were identified namely, } \\
\text { Aspergillus ( } 17 \text { species), Penicillium ( } 7 \text { species), Alternaria, Curvularia, } \\
\text { Fusarium ( } 2 \text { species each), Acrophialophora, Botryotrichum, Choanephora, } \\
\text { Helminthosporium, Mammaria, Trichocladium, Trichoderma, Trichurus and } \\
\text { Verticillium ( } 1 \text { species each). The most common species isolated was A. niger } \\
\text { followed by } P \text {. javanicum, Alternaria humicola, A. sulphureus, Fusarium } \\
\text { moniliforme, P. funiculosum, P. nigricans, P. spinulosum, Trichoderma } \\
\text { polysporum and Trichurus spiralis. From the present study, it appears that the } \\
\text { coastal soils contain a mycoflora reservoir comprising of a variety of genera } \\
\text { which contributes significantly to the ecological functioning of a marine } \\
\text { ecosystem. }\end{array}$ \\
\hline
\end{tabular}

\section{Introduction}

In benthic marine environments, marine fungi represent ecologically important group amongst benthic organisms, acting as key intermediates of energy flow from detritus to higher trophic levels in marine ecosystems (Kohlmeyer and Kohlmeyer, 1979; Hyde et al., 2000). The ecological importance of filamentous fungi in marine systems is underestimated; these organisms represent a diverse range of saprobes, pathogens and symbionts forming an integral part of coastal and deep-sea environments (Hyde et al., 1998; Bass et al., 2007).

Marine mycology has grown into a significant mycological subdiscipline during the last century with mycologists reporting the occurrence of marine fungi as early as 1849 (Jones et al., 2009). By 1996, diversity estimates of marine fungi were placed at 
around 1500 species, and by 2011, estimates projected the number of possible marine fungi as over 10,000 species (Jones, 2011). Marine fungi are considered important ecological components in marine environments due to their performance in biogeochemical processes (Tedersoo et al., 2014; 2015).

A great diversity of fungi has been reported from along coastlines, such as mangrove, sand, beach and estuarine habitats (Morrison-Gardiner, 2002; Menezes et al., 2010; Behera et al., 2012). Marine fungi are classified into obligate and facultative forms: obligate marine fungi are those that grow and sporulate exclusively in a marine or estuarine habitat, and facultative marine fungi are those that also occur in freshwater or terrestrial milieus yet are able to grow in, and frequently are isolated from marine habitats (Kohlmeyer and Kohlmeyer, 1979).

Fungi are particularly diverse and abundant in soils. Most of the studies on soil mycoflora in coastal areas have been conducted by researchers in Greece (Papadakis et al., 1997), Italy (Mancini et al., 2005), Spain (Larrondo and Calvo, 1989), Egypt (Fatma, 2003), Brazil (Gomes et al., 2008), Malaysia (Zakaria et al., 2011) and India (Behera et al., 2012; Anitha and Nayak, 2014; Ashok et al., 2015). Nevertheless, in India, knowledge on soil fungi in coastal area is still lacking. Therefore, this study was carried out in order to observe the diversity of soil fungi in coastal area of three different locations of Tuticorin Dt.

\section{Materials and Methods}

\section{Study Site}

Tuticorin Coast is located in the south eastern part of coastal zone of Tamil Nadu State, India. It lies between $8^{\circ} 41^{\prime} 49^{\prime \prime} \mathrm{N}$ and
9'22'20' N latitudes and 78'3'56' $\mathrm{E}$ and 79²6'6”' E longitudes.

\section{Collection of Soil Samples}

The soil samples were collected seasonally from three different coastal areas such as Palayakayal, Punnakayal and Tuticorin. The soil samples were collected at a depth within $10 \mathrm{~cm}$ using a metal spatula. The spatula was sterilized every time with 70 per cent alcohol. At each station 5 to 7 samples were collected randomly and were pooled together. The samples were kept in sterilized polythene bags, sealed and transported to the laboratory.

\section{Isolation of Fungi}

The soil mycoflora were isolated by the method of Warcup (1950). Soil sample weighed $1 \mathrm{~g}$ was diluted in $10 \mathrm{ml}$ of $50 \%$ seawater $(1: 1 \mathrm{v} / \mathrm{v}$ seawater $(30 \mathrm{ppt})$ : distilled water). One $\mathrm{ml}$ of the diluted sample $\left(10^{-2}\right.$ and $10^{-3}$ ) was poured and spreaded on the petriplates containing sterilized PDA medium (Potato - 200 gms, dextrose -20

gms, agar $\quad-15 \mathrm{gms}$, distilled water -500 $\mathrm{ml}$, sea water - 500ml, $\mathrm{pH}-6.5$ ) supplemented with one percent streptomycin sulphate solution for preventing bacterial growth in replicates. The inoculated plates were kept in the aseptic conditions at the room temperature $\left(24 \pm 2^{\circ} \mathrm{C}\right)$ for 3 - 5 days. The colonies growing on PDA plates with different morphology were counted separately. The fungal cultures were then transferred, subcultured and the pure cultures were maintained on PDA medium.

Fungal morphology were studied macroscopically by observing colour and texture and microscopically by staining with lactophenol cotton blue and observed under Nikon phase contrast microscope (Nikon, Japan) for the conidia, conidiophores and arrangement of spores. 


\section{Identification}

The identification of the fungal taxa was done using the standard manuals of fungi such as A Manual of Penicillia (Raper and Thom, 1949), A Manual of Soil fungi (Gillman, 1957), The genus Aspergillus (Raper and Fennell, 1965), Hyphomycetes (Subramanian, 1971), Dematiaceous Hyphomycetes (Ellis, 1971, 1976), Marine Mycology The Higher fungi (Kohlmeyer and Kohlmeyer, 1979) and Compendium of soil fungi (Domsch et al., 1980).

\section{Presentation of Data}

Percentage of contribution and percentage of frequency of fungal isolates were calculated by using the following formulas.

No. of colonies of fungus in a sample

Total number all colonies of all the species in a sample
}

Number of samples in which a particular fungus occurred

$\%$ frequency $=\underset{\text { Total number of samples examined }}{\mathrm{x}} 100$

Based on the frequency occurrences the fungi were grouped as rare (0-25\% frequency), occasional (26-50\% frequency), frequent (51-75\% frequency) and common (76-100\% frequency) species.

\section{Physico-chemical Analysis of Soil}

The physico-chemical parameters of collected soil samples were analyzed by APHA method (1989) in Soil Testing Laboratory, Department of Agriculture, Government of Tamil Nadu, Tiruchirappalli20.

\section{Statistical Analysis}

The relationship between the physicochemical parameters and total fungal colonies was studied by Pearson's correlation method. The data were computed and analyzed using Statistical Package for Social Sciences (SPSS) software.

\section{Results and Discussion}

The occurrence of marine fungi has been reported in different substrates such as sponges, algae, wood, tunicates, sediments, mollusks, corals, plants, fish and the ecology and phylogeny of this group were summarized (Jones, 2000; Jones et al., 2009 \& 2011; Jones and Pang, 2012; Richards et al., 2012).

In the present study, a total of 39 marine fungal species were recorded (Table 1). Based on morphological characteristics, fourteen genera of mycoflora were identified namely, Aspergillus (17 species), Penicillium (7 species), Alternaria, Curvularia, Fusarium (2 species each), Acrophialophora, Choanephora, Botryotrichum, Mammaria, Trichurus and Verticillium (1 species each). In the same way, Raveendran and Manimohan (2007) reported maximum number of marine fungi (72) from Kerala and Prasannarai and Sridhar (1993) reported 32 taxa from Mangalore coast.

The maximum fungal species were isolated from Punnakayal station followed by Tuticorin and Palayakayal (Table 2-4).

Similarly, the taxonomic and ecological studies of marine fungi from Maharashtra (Patil and Borse, 1983), Karnataka (Prasannarai and Sridhar, 1993), Tamil Nadu (Ravikumar and Vittal, 1996), Goa (Borse et al., 1999), Gujarat (Borse et al., 2000), West Bengal (Borse and Pawar, 2001) and Andhra Pradesh (Sarma and Vittal, 2001) have been reported in various part of the India. 


\section{Int.J.Curr.Res.Aca.Rev.2016; 4(2): $x x-x x$}

Table.1 List of Isolated Soil Mycoflora from Coastal Area of Tuticorin dt. In the Year of 2013-2014

\begin{tabular}{|l|l|}
\hline S. No. & Name of the isolates \\
\hline 1. & Acrophialophora fusispora \\
\hline 2. & Alternaria geophila \\
\hline 3. & A. humicola \\
\hline 4. & Aspergillus candidus \\
\hline 5. & A. conicus \\
\hline 6. & A. flavus \\
\hline 7. & A. fuscus \\
\hline 8. & A.granulosus \\
\hline 9. & A. niger \\
\hline 10. & A. ochraceus \\
\hline 11. & A. parvulus \\
\hline 12. & A. phoenicis \\
\hline 13. & A. repens \\
\hline 14. & A.rugulosus \\
\hline 15. & A. sulphureus \\
\hline 16. & A. terreus \\
\hline 17. & A. terricola \\
\hline 18. & A. ellipticus \\
\hline 19. & A. thomii \\
\hline 20. & A. unguis \\
\hline 21. & Botryotrichum atrogriseum \\
\hline 22. & Choanephora cucurbitarum \\
\hline 23. & Curvularia geniculata \\
\hline 24. & Curvularia sp. \\
\hline 25. & Helminthosporium oryzae \\
\hline 26. & Fusarium moniliforme \\
\hline 27. & F. equiseti \\
\hline 28. & Mammaria echinobotryoides \\
\hline 29. & Penicillium granulatum \\
\hline 30. & P. janthinellum \\
\hline 31. & P. funiculosum \\
\hline 32. & P. javanicum \\
\hline 33. & P. nigricans \\
\hline 34. & P. rubrum \\
\hline 35. & $P$. spinulosum \\
\hline 36. & Trichocladium opacum \\
\hline 37. & Trichoderma polysporum \\
\hline 38. & Trichurus spiralis \\
\hline 39. & Verticillium glaucum \\
\hline & \\
\hline
\end{tabular}




\section{Int.J.Curr.Res.Aca.Rev.2016; 4(2): $x x-x x$}

Table.2 Total Number of Colonies, Mean Density $(\mathrm{Cfu} / \mathrm{G})$ and Percentage Contribution of Fungi from Palayakayal in the Year of 2013-2014

\begin{tabular}{|c|c|c|c|c|c|c|c|c|c|c|c|}
\hline \multirow{2}{*}{ S. No. } & \multirow{2}{*}{ Name of the organisms } & \multicolumn{2}{|c|}{ Post monsoon } & \multicolumn{2}{|c|}{ Summer } & \multicolumn{2}{|c|}{ Pre monsoon } & \multicolumn{2}{|c|}{ Monsoon } & \multirow{2}{*}{$\begin{array}{c}\text { Total no. of } \\
\text { colonies }\end{array}$} & \multirow{2}{*}{$\begin{array}{c}\% \text { of } \\
\text { contribution }\end{array}$} \\
\hline & & TNC & MD & TNC & MD & TNC & MD & TNC & MD & & \\
\hline 1 & Aspergillus fuscus & 1 & 0.33 & 1 & 0.33 & 2 & 0.67 & - & - & 4 & 3.17 \\
\hline 2 & A. niger & 1 & 0.33 & 4 & 1.33 & 4 & 1.33 & 4 & 1.33 & 13 & 10.32 \\
\hline 3 & A. parvulus & - & - & 4 & 1.33 & 2 & 0.67 & - & - & 6 & 4.76 \\
\hline 4 & A. repens & 3 & 1.00 & - & - & 2 & 0.67 & 3 & 1.00 & 8 & 6.35 \\
\hline 5 & A. sulphureus & 3 & 1.00 & - & - & 3 & 1.00 & 3 & 1.00 & 9 & 7.14 \\
\hline 6 & Botryotrichum atrogriseum & - & - & 6 & 2.00 & 6 & 2.00 & - & - & 12 & 9.52 \\
\hline 7 & Choanephora cucurbitarum & 4 & 1.33 & - & - & - & - & - & - & 4 & 3.17 \\
\hline 8 & Curvularia geniculata & - & - & 3 & 1.00 & 3 & 1.00 & - & - & 6 & 4.76 \\
\hline 9 & Fusarium moniliforme & 3 & 1.00 & 3 & 1.00 & 3 & 1.00 & 3 & 1.00 & 12 & 9.52 \\
\hline 10 & Fusarium equiseti & 2 & 0.67 & - & - & 3 & 1.00 & - & - & 5 & 3.97 \\
\hline 11 & Penicillium funiculosum & 3 & 1.00 & - & - & - & - & 2 & 0.67 & 5 & 3.97 \\
\hline 12 & P. granulatum & 1 & 0.33 & - & - & 3 & 1.00 & 2 & 0.67 & 6 & 4.76 \\
\hline 13 & P. javanicum & 4 & 1.33 & 2 & 0.67 & 2 & 0.67 & 2 & 0.67 & 10 & 7.94 \\
\hline 14 & P. janthinellum & 4 & 1.33 & - & - & 2 & 0.67 & - & - & 6 & 4.76 \\
\hline 15 & P. nigricans & 1 & 0.33 & 1 & 0.33 & - & - & 4 & 1.33 & 6 & 4.76 \\
\hline 16 & P. rubrum & 1 & 0.33 & 1 & 0.33 & 4 & 1.33 & - & - & 6 & 4.76 \\
\hline \multirow[t]{2}{*}{17} & P. spinulosum & - & - & - & - & 4 & 1.33 & 4 & 1.33 & 8 & 6.35 \\
\hline & & 31.00 & 10.33 & 25.00 & 8.33 & 43.00 & 14.33 & 27.00 & 9.00 & 126.00 & 100 \\
\hline
\end{tabular}

TNC - Total Number of Colonies; MD - Mean Density 
Int.J.Curr.Res.Aca.Rev.2016; 4(2): $x x-x x$

Table.3 Total Number of Colonies, Mean Density (cfu/g) and Percentage Contribution of Fungi from Punnakayal in the Year of 2013-2014

\begin{tabular}{|c|c|c|c|c|c|c|c|c|c|c|c|}
\hline \multirow{2}{*}{$\begin{array}{l}\text { S. } \\
\text { No. }\end{array}$} & \multirow[t]{2}{*}{ Name of the organisms } & \multicolumn{2}{|c|}{$\begin{array}{c}\text { Post } \\
\text { monsoon }\end{array}$} & \multicolumn{2}{|c|}{ Summer } & \multicolumn{2}{|c|}{ Pre monsoon } & \multicolumn{2}{|c|}{ Monsoon } & \multirow{2}{*}{$\begin{array}{l}\text { Total no. of } \\
\text { colonies }\end{array}$} & \multirow[t]{2}{*}{$\%$ of contribution } \\
\hline & & TNC & MD & TNC & MD & TNC & MD & TNC & MD & & \\
\hline 1 & Alternaria humicola & 1 & 0.33 & 2 & 0.67 & 2 & 0.67 & 1 & 0.33 & 6 & 4.23 \\
\hline 2 & Aspergillus candidus & - & - & 2 & 0.67 & - & - & 2 & 0.67 & 4 & 2.82 \\
\hline 3 & A. conicus & 4 & 1.33 & - & - & - & - & 6 & 2.00 & 10 & 7.04 \\
\hline 4 & A.granulosus & - & - & - & - & 3 & 1.00 & 1 & 0.33 & 4 & 2.82 \\
\hline 5 & A. niger & 4 & 1.33 & 4 & 1.33 & 4 & 1.33 & 1 & 0.33 & 13 & 9.15 \\
\hline 6 & A. rugulosus & 3 & 1.00 & - & - & 3 & 1.00 & - & - & 6 & 4.23 \\
\hline 7 & A. terricola & - & - & - & - & 4 & 1.33 & 4 & 1.33 & 8 & 5.63 \\
\hline 8 & A. thomii & 2 & 0.67 & - & - & 2 & 0.67 & 2 & 0.67 & 6 & 4.23 \\
\hline 9 & A. unguis & 1 & 0.33 & 1 & 0.33 & - & - & 3 & 1.00 & 5 & 3.52 \\
\hline 10 & Curvularia sp. & - & - & 1 & 0.33 & 1 & 0.33 & 1 & 0.33 & 3 & 2.11 \\
\hline 11 & Helminthosporium oryzae & 4 & 1.33 & - & - & 3 & 1.00 & - & - & 7 & 4.93 \\
\hline 12 & Mammaria echinobotryoides & 4 & 1.33 & - & - & 4 & 1.33 & 4 & 1.33 & 12 & 8.45 \\
\hline 13 & P. granulatum & - & - & - & - & 1 & 0.33 & 4 & 1.33 & 5 & 3.52 \\
\hline 14 & Penicillium janthinellum & - & - & 3 & 1.00 & 3 & 1.00 & 3 & 1.00 & 9 & 6.34 \\
\hline 15 & $P$. javanicum & 4 & 1.33 & - & - & - & - & 4 & 1.33 & 8 & 5.63 \\
\hline 16 & $P$ funiculosum & 2 & 0.67 & - & - & 2 & 0.67 & 2 & 0.67 & 6 & 4.23 \\
\hline 17 & P. nigricans & 2 & 0.67 & 2 & 0.67 & 2 & 0.67 & 2 & 0.67 & 8 & 5.63 \\
\hline 18 & P. rubrum & 1 & 0.33 & 1 & 0.33 & 2 & 0.67 & - & - & 4 & 2.82 \\
\hline 19 & P. spinulosum & 2 & 0.67 & 2 & 0.67 & 2 & 0.67 & 2 & 0.67 & 8 & 5.63 \\
\hline 20 & Trichoderma polysporum & 2 & 0.67 & 2 & 0.67 & 4 & 1.33 & 2 & 0.67 & 10 & 7.04 \\
\hline & & 36 & 12 & 20 & 6.67 & 42 & 14 & 44 & 14.67 & 142 & 100 \\
\hline
\end{tabular}

TNC - Total Number of Colonies; MD - Mean Density 


\section{Int.J.Curr.Res.Aca.Rev.2016; 4(2): $x x-x x$}

Table.4 Total Number of Colonies, Mean Density (CFU/g) and Percentage Contribution of Fungi from Tuticorin in the Year of $2013-2014$

\begin{tabular}{|c|c|c|c|c|c|c|c|c|c|c|c|}
\hline \multirow{2}{*}{$\begin{array}{l}\text { S. } \\
\text { No. }\end{array}$} & \multirow{2}{*}{ Name of the organisms } & \multicolumn{2}{|c|}{$\begin{array}{c}\text { Post } \\
\text { monsoon }\end{array}$} & \multicolumn{2}{|c|}{ Summer } & \multicolumn{2}{|c|}{ Pre monsoon } & \multicolumn{2}{|c|}{ Monsoon } & \multirow{2}{*}{$\begin{array}{l}\text { Total no. of } \\
\text { colonies }\end{array}$} & \multirow{2}{*}{$\begin{array}{c}\% \text { of } \\
\text { contribution }\end{array}$} \\
\hline & & TNC & MD & TNC & MD & TNC & MD & TNC & MD & & \\
\hline 1 & Acrophialophora fusispora & - & - & 2 & 0.67 & - & - & - & - & 2 & 1.87 \\
\hline 2 & Alternaria geophila & - & - & 1 & 0.33 & 1 & 0.33 & - & - & 2 & 1.87 \\
\hline 3 & Aspergillus ellipticus & - & - & 2 & 0.67 & - & - & 2 & 0.67 & 4 & 3.74 \\
\hline 4 & A. flavus & 3 & 1.00 & - & - & 2 & 0.67 & - & - & 5 & 4.67 \\
\hline 5 & A. niger & 1 & 0.33 & 4 & 1.33 & 1 & 0.33 & 1 & 0.33 & 7 & 6.54 \\
\hline 6 & A. ochraceus & 2 & 0.67 & - & - & 2 & 0.67 & 3 & 1.00 & 7 & 6.54 \\
\hline 7 & A. phoenicis & 2 & 0.67 & - & - & 2 & 0.67 & 2 & 0.67 & 6 & 5.61 \\
\hline 8 & A. terreus & 1 & 0.33 & - & - & 1 & 0.33 & 1 & 0.33 & 3 & 2.80 \\
\hline 9 & Penicillium funiculosum & 3 & 1.00 & 1 & 0.33 & 2 & 0.67 & 2 & 0.67 & 8 & 7.48 \\
\hline 10 & P. granulatum & 4 & 1.33 & - & - & 4 & 1.33 & 2 & 0.67 & 10 & 9.35 \\
\hline 11 & P. janthinellum & 2 & 0.67 & - & - & 2 & 0.67 & 2 & 0.67 & 6 & 5.61 \\
\hline 12 & P. javanicum & 2 & 0.67 & 1 & 0.33 & 2 & 0.67 & 3 & 1.00 & 8 & 7.48 \\
\hline 13 & P. nigricans & - & - & 3 & 1.00 & - & - & 3 & 1.00 & 6 & 5.61 \\
\hline 14 & P. rubrum & - & - & 3 & 1.00 & - & - & 4 & 1.33 & 7 & 6.54 \\
\hline 15 & P. spinulosum & 1 & 0.33 & 1 & 0.33 & 2 & 0.67 & - & - & 4 & 3.74 \\
\hline 16 & Trichoderma opacum & 4 & 1.33 & - & - & 4 & 1.33 & - & - & 8 & 7.48 \\
\hline 17 & Trichurus spiralis & 3 & 1.00 & 3 & 1.00 & 3 & 1.00 & 1 & 0.33 & 10 & 9.35 \\
\hline \multirow[t]{2}{*}{18} & Verticillium glaucum & - & - & - & - & - & - & 4 & 1.33 & 4 & 3.74 \\
\hline & & 28 & 9.33 & 21 & 7 & 28 & 9.33 & 30 & 10 & 107 & 100 \\
\hline
\end{tabular}

TNC - Total Number of Colonies; MD - Mean Density 


\section{Int.J.Curr.Res.Aca.Rev.2016; 4(2): $x x-x x$}

Table.5 Percentage Frequency and Frequency Class of Different Species of Fungi Recorded at Three Stations $(\mathrm{N}=24)$ in the Year of 2013-2014

\begin{tabular}{|c|c|c|c|c|c|c|c|c|c|c|}
\hline \multirow[b]{2}{*}{$\begin{array}{l}\text { S. } \\
\text { No. }\end{array}$} & \multirow[b]{2}{*}{$\begin{array}{l}\text { Name of the } \\
\text { organisms }\end{array}$} & \multicolumn{3}{|c|}{ Palayakayal } & \multicolumn{3}{|c|}{ Punnakayal } & \multicolumn{3}{|l|}{ Tuticorin } \\
\hline & & 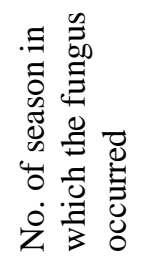 & 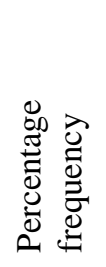 & 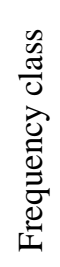 & 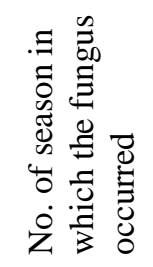 & 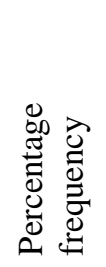 & 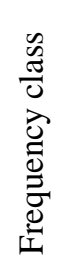 & 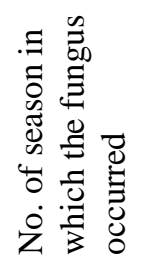 & 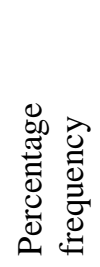 & 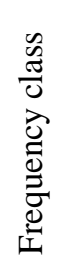 \\
\hline 1. & $\begin{array}{l}\text { Acrophialophora } \\
\text { fusispora }\end{array}$ & & & & & & & 1 & 25 & $\mathrm{R}$ \\
\hline 2. & Alternaria geophila & & & & & & & 2 & 50 & $\mathrm{O}$ \\
\hline 3. & A. humicola & & & & 4 & 100 & $\mathrm{C}$ & & & \\
\hline 4. & Aspergillus candidus & & & & 2 & 50 & $\mathrm{O}$ & & & \\
\hline 5. & A. conicus & & & & 2 & 50 & $\mathrm{O}$ & & & \\
\hline 6. & A. ellipticus & & & & & & & 2 & 50 & $\mathrm{O}$ \\
\hline 7. & A. flavus & & & & & & & 2 & 50 & $\mathrm{O}$ \\
\hline 8. & A. fuscus & 3 & 75 & $\mathrm{~F}$ & & & & & & \\
\hline 9. & A.granulosus & & & & 2 & 50 & $\mathrm{O}$ & & & \\
\hline 10. & A. niger & 4 & 100 & $\mathrm{C}$ & 4 & 100 & $\mathrm{C}$ & 4 & 100 & $\mathrm{C}$ \\
\hline 11. & A. ochraceus & & & & & & & 3 & 75 & $\mathrm{~F}$ \\
\hline 12. & A. parvulus & 2 & 50 & $\mathrm{O}$ & & & & & & \\
\hline 13. & A. phoenicis & & & & & & & 3 & 75 & $\mathrm{~F}$ \\
\hline 14. & A. repens & 3 & 75 & $\mathrm{~F}$ & & & & & & \\
\hline 15. & A. rugulosus & & & & 2 & 50 & $\mathrm{O}$ & & & \\
\hline 16. & A. sulphureus & 3 & 75 & $\mathrm{C}$ & & & & & & \\
\hline 17. & A. terreus & & & & & & & 3 & 75 & $\mathrm{~F}$ \\
\hline 18. & A. terricola & & & & 2 & 50 & $\mathrm{O}$ & & & \\
\hline 19. & A. thomii & & & & 3 & 75 & $\mathrm{~F}$ & & & \\
\hline 20. & A. unguis & & & & 3 & 75 & $\mathrm{~F}$ & & & \\
\hline 21. & $\begin{array}{l}\text { Botryotrichum } \\
\text { atrogriseum }\end{array}$ & 2 & 50 & $\mathrm{O}$ & & & & & & \\
\hline 22. & $\begin{array}{l}\text { Choanephora } \\
\text { cucurbitarum }\end{array}$ & 1 & 25 & $\mathrm{R}$ & & & & & & \\
\hline 23. & Curvularia. sp & & & & 3 & 75 & $\mathrm{~F}$ & & & \\
\hline
\end{tabular}


Int.J.Curr.Res.Aca.Rev.2016; 4(2): $x x-x x$

\begin{tabular}{|c|c|c|c|c|c|c|c|c|c|c|}
\hline 24. & $\begin{array}{l}\text { Curvularia } \\
\text { geniculata }\end{array}$ & 2 & 50 & $\mathrm{O}$ & & & & & & \\
\hline 25. & $\begin{array}{l}\text { Helminthosporium } \\
\text { oryzae }\end{array}$ & & & & 2 & 50 & 0 & & & \\
\hline 26. & $\begin{array}{l}\text { Fusarium } \\
\text { moniliforme }\end{array}$ & 4 & 100 & $\mathrm{C}$ & & & & & & \\
\hline 27. & F. equiseti & 2 & 50 & $\mathrm{O}$ & & & & & & \\
\hline 28. & $\begin{array}{l}\text { Mammaria } \\
\text { echinobotryoides }\end{array}$ & & & & 3 & 75 & $\mathrm{~F}$ & & & \\
\hline 29. & $\begin{array}{l}\text { Penicillium } \\
\text { granulatum }\end{array}$ & 3 & 75 & $\mathrm{~F}$ & 2 & 50 & $\mathrm{O}$ & 3 & 75 & $\mathrm{~F}$ \\
\hline 30. & P. janthinellum & 2 & 50 & $\mathrm{O}$ & 3 & 75 & $\mathrm{~F}$ & 3 & 75 & $\mathrm{~F}$ \\
\hline 31. & P. funiculosum & 2 & 50 & $\mathrm{O}$ & 3 & 75 & $\mathrm{~F}$ & 4 & 100 & $\mathrm{C}$ \\
\hline 32. & P. javanicum & 4 & 100 & $\mathrm{C}$ & 3 & 75 & $\mathrm{~F}$ & 4 & 100 & $\mathrm{C}$ \\
\hline 33 & P. nigricans & 3 & 75 & $\mathrm{~F}$ & 4 & 100 & $\mathrm{C}$ & 2 & 50 & $\mathrm{O}$ \\
\hline 34 & P. rubrum & 3 & 75 & $\mathrm{~F}$ & 3 & 75 & $\mathrm{~F}$ & 2 & 50 & $\mathrm{O}$ \\
\hline 35 & P. spinulosum & 2 & 50 & $\mathrm{O}$ & 4 & 100 & $\mathrm{C}$ & 2 & 50 & $\mathrm{O}$ \\
\hline 36 & $\begin{array}{l}\text { Trichocladium } \\
\text { opacum }\end{array}$ & & & & & & & 2 & 50 & $\mathrm{O}$ \\
\hline 37 & $\begin{array}{l}\text { Trichoderma } \\
\text { polysporum }\end{array}$ & & & & 4 & 100 & $\mathrm{C}$ & & & \\
\hline 38 & Trichurus spiralis & & & & & & & 4 & 100 & $\mathrm{C}$ \\
\hline 39 & Verticillium glaucum & & & & & & & 1 & 25 & $\mathrm{R}$ \\
\hline
\end{tabular}

$\mathrm{R}$ - Rare (0-25\%); O - Occasional (26-50\%); F - Frequent (51-75\%); C - Common (76-100\%) 
Int.J.Curr.Res.Aca.Rev.2016; 4(2): $x x-x x$

Table.6 Physico - Chemical Characteristics of the Soil Samples Collected from Palayakayal

\begin{tabular}{|c|c|c|c|c|c|}
\hline \multirow[t]{2}{*}{ S. No. } & \multirow[t]{2}{*}{ Name of the parameters } & \multicolumn{4}{|c|}{ Palayakayal (2013-2014) } \\
\hline & & $\begin{array}{c}\text { Post } \\
\text { monsoon }\end{array}$ & Summer & Pre monsoon & Monsoon \\
\hline 1 & $\mathrm{pH}$ & 8.51 & 8.63 & 8.22 & 8.33 \\
\hline 2 & Salinity & 31 & 33 & 30 & 29 \\
\hline 3 & $\mathrm{EC}\left(\mathrm{dsm}^{-1}\right)$ & 2.33 & 2.47 & 2.39 & 2.47 \\
\hline 4 & Organic Carbon (\%) & 0.34 & 0.33 & 0.39 & 0.40 \\
\hline 5 & Organic Matter (\%) & 0.59 & 0.52 & 0.61 & 0.60 \\
\hline 6 & Available Nitrogen (\%) & 0.968 & 0.701 & 0.833 & 0.779 \\
\hline 7 & Available Phosphorus (\%) & 0.294 & 0.268 & 0.213 & 0.233 \\
\hline 8 & Available Potassium (\%) & 0.950 & 0.991 & 0.913 & 0.901 \\
\hline 9 & Available Zinc (ppm) & 0.91 & 0.95 & 1.51 & 1.99 \\
\hline 10 & Available Copper (ppm) & 0.98 & 0.90 & 0.70 & 0.88 \\
\hline 11 & Available Iron (ppm) & 4.99 & 4.71 & 4.63 & 4.73 \\
\hline 12 & Available Manganese (ppm) & 2.15 & 2.19 & 2.54 & 2.58 \\
\hline 13 & $\begin{array}{l}\text { Cat ion exchange capacity (C. Mole } \\
\text { Proton }^{+} / \mathrm{kg} \text { ) }\end{array}$ & 21.12 & 22.6 & 21.7 & 22.3 \\
\hline 14 & Calcium (C. Mole Proton ${ }^{+} / \mathrm{kg}$ ) & 19.1 & 20.3 & 17.5 & 14.6 \\
\hline 15 & 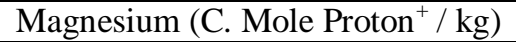 & 8.1 & 8.6 & 7.9 & 8.2 \\
\hline 16 & Sodium (C. Mole Proton ${ }^{+} / \mathrm{kg}$ ) & 1.99 & 2.01 & 1.51 & 1.63 \\
\hline 17 & Potassium (C. Mole Proton ${ }^{+} / \mathrm{kg}$ ) & 0.21 & 0.25 & 0.24 & 0.23 \\
\hline & Total fungal colonies & 31 & 25 & 43 & 27 \\
\hline
\end{tabular}

Table.7 Physico - Chemical Characteristics of the Soil Samples Collected from Punnakayal

\begin{tabular}{|c|c|c|c|c|c|}
\hline \multirow[b]{2}{*}{ S. No. } & \multirow[b]{2}{*}{ Name of the parameters } & \multicolumn{4}{|c|}{ Punnakayal (2013-2014) } \\
\hline & & $\begin{array}{c}\text { Post } \\
\text { monsoon }\end{array}$ & Summer & Pre monsoon & Monsoon \\
\hline 1 & $\mathrm{pH}$ & 8.57 & 8.63 & 8.39 & 8.42 \\
\hline 2 & Salinity & 30 & 31 & 30 & 29 \\
\hline 3 & $\mathrm{EC}\left(\mathrm{dsm}^{-1}\right)$ & 1.91 & 1.99 & 1.89 & 1.95 \\
\hline 4 & Organic Carbon (\%) & 0.44 & 0.51 & 0.64 & 0.63 \\
\hline 5 & Organic Matter (\%) & 0.32 & 0.31 & 0.39 & 0.37 \\
\hline 6 & Available Nitrogen (\%) & 0.913 & 0.859 & 0.989 & 0.769 \\
\hline 7 & Available Phosphorus (\%) & 0.251 & 0.261 & 0.226 & 0.262 \\
\hline 8 & Available Potassium (\%) & 0.852 & 0.896 & 0.801 & 0.788 \\
\hline 9 & Available Zinc (ppm) & 1.33 & 1.59 & 1.51 & 1.71 \\
\hline 10 & Available Copper (ppm) & 0.97 & 0.82 & 0.72 & 0.95 \\
\hline 11 & Available Iron (ppm) & 4.61 & 4.88 & 4.67 & 4.51 \\
\hline 12 & Available Manganese (ppm) & 2.96 & 2.88 & 2.99 & 2.76 \\
\hline 13 & $\begin{array}{l}\text { Cat ion exchange capacity (C. Mole } \\
\text { Proton }^{+} / \mathrm{kg} \text { ) }\end{array}$ & 21.7 & 24.1 & 25.1 & 26.1 \\
\hline 14 & Calcium (C. Mole Proton ${ }^{+} / \mathrm{kg}$ ) & 17.1 & 19.1 & 16.5 & 17.7 \\
\hline 15 & Magnesium(C. Mole Proton ${ }^{+} / \mathrm{kg}$ ) & 8.2 & 8.8 & 8.0 & 9.0 \\
\hline 16 & Sodium (C. Mole Proton ${ }^{+} / \mathrm{kg}$ ) & 1.89 & 1.98 & 1.33 & 1.61 \\
\hline 17 & Potassium (C. Mole Proton ${ }^{+} / \mathrm{kg}$ ) & 0.25 & 0.29 & 0.24 & 0.25 \\
\hline & Total fungal colonies & 36 & 20 & 42 & 44 \\
\hline
\end{tabular}




\section{Int.J.Curr.Res.Aca.Rev.2016; 4(2): $x x-x x$}

Table.8 Physico - Chemical Characteristics of the Soil Samples Collected from Tuticorin

\begin{tabular}{|c|c|c|c|c|c|}
\hline \multirow[b]{2}{*}{ S. No. } & \multirow[b]{2}{*}{ Name of the parameters } & \multicolumn{4}{|c|}{ Tuticorin (2013-2014) } \\
\hline & & Post monsoon & Summer & $\begin{array}{c}\text { Pre } \\
\text { monsoon }\end{array}$ & Monsoon \\
\hline 1 & $\mathrm{pH}$ & 8.46 & 8.72 & 8.23 & 8.32 \\
\hline 2 & Salinity & 32 & 36 & 30 & 31 \\
\hline 3 & $\mathrm{EC}\left(\mathrm{dsm}^{-1}\right)$ & 2.43 & 2.79 & 2.27 & 2.15 \\
\hline 4 & Organic Carbon (\%) & 0.39 & 0.27 & 0.45 & 0.29 \\
\hline 5 & Organic Matter (\%) & 0.52 & 0.45 & 0.63 & 0.71 \\
\hline 6 & Available Nitrogen $(\%)$ & 0.894 & 0.869 & 0.915 & 0.745 \\
\hline 7 & Available Phosphorus (\%) & 0.257 & 0.227 & 0.295 & 0.256 \\
\hline 8 & Available Potassium (\%) & 0.999 & 0.993 & 0.818 & 0.869 \\
\hline 9 & Available Zinc (ppm) & 1.09 & 1.32 & 1.65 & 1.72 \\
\hline 10 & Available Copper (ppm) & 0.62 & 0.80 & 0.94 & 0.63 \\
\hline 11 & Available Iron (ppm) & 4.26 & 5.01 & 5.26 & 4.96 \\
\hline 12 & Available Manganese (ppm) & 3.52 & 2.62 & 2.74 & 1.85 \\
\hline 13 & $\begin{array}{l}\text { Cat ion exchange capacity (C. Mole } \\
\text { Proton }^{+} / \mathrm{kg} \text { ) }\end{array}$ & 29.9 & 26.1 & 23.9 & 27.7 \\
\hline 14 & Calcium(C. Mole Proton ${ }^{+} / \mathrm{kg}$ ) & 15.7 & 17.6 & 15.3 & 15.9 \\
\hline 15 & Magnesium(C. Mole Proton $\left.{ }^{+} / \mathrm{kg}\right)$ & 8.1 & 8.5 & 7.9 & 7.6 \\
\hline 16 & Sodium(C. Mole Proton $\left.{ }^{+} / \mathrm{kg}\right)$ & 1.33 & 1.44 & 1.33 & 1.33 \\
\hline 17 & Potassium(C. Mole Proton $^{+} / \mathrm{kg}$ ) & 0.23 & 0.33 & 0.27 & 0.24 \\
\hline & Total fungal colonies & 28 & 21 & 28 & 30 \\
\hline
\end{tabular}


Int.J.Curr.Res.Aca.Rev.2016; 4(2): $x x-x x$

Table.9 Correlation of Physico - Chemical and Total Number of Colony Characteristics of the

Soil Samples of Palayakayal in the Year of 2013 -2014

\begin{tabular}{|c|c|c|c|c|c|c|c|c|c|c|c|c|c|c|c|c|c|c|}
\hline & PH & SA & EC & OC & OM & $\mathbf{A N}$ & AP & AK & $\mathbf{A Z}$ & $\mathbf{A C}$ & AI & $\mathbf{A M}$ & CEC & $\mathbf{C A}$ & MG & NA & $\mathbf{K}$ & TNC \\
\hline $\mathbf{P H}$ & 1 & & & & & & & & & & & & & & & & & \\
\hline SA & $0.989^{*}$ & 1 & & & & & & & & & & & & & & & & \\
\hline EC & $\mathbf{0 . 9 3 9}$ & $\mathbf{0 . 9 3 9}$ & 1 & & & & & & & & & & & & & & & \\
\hline OC & -0.651 & -0.687 & -0.396 & 1 & & & & & & & & & & & & & & \\
\hline OM & -0.863 & -0.822 & $\mathbf{0 . 9 5 0}^{*}$ & 0.177 & 1 & & & & & & & & & & & & & \\
\hline $\mathbf{A N}$ & 0.109 & 0.075 & 0.414 & 0.672 & -0.586 & 1 & & & & & & & & & & & & \\
\hline AP & -0.906 & -0.894 & -0.708 & 0.882 & 0.590 & 0.308 & 1 & & & & & & & & & & & \\
\hline$\overline{\mathbf{A K}}$ & 0.854 & 0.766 & 0.753 & $\begin{array}{l} \\
0.426\end{array}$ & -0.829 & 0.174 & -0.794 & 1 & & & & & & & & & & \\
\hline $\mathbf{A Z}$ & -0.627 & -0.511 & -0.631 & $\begin{array}{l}- \\
0.003 \\
\end{array}$ & 0.822 & $\begin{array}{l}- \\
0.499\end{array}$ & 0.458 & -0.903 & 1 & & & & & & & & & \\
\hline $\mathbf{A C}$ & -0.145 & -0.048 & 0.165 & 0.438 & -0.091 & 0.580 & 0.459 & -0.480 & 0.341 & 1 & & & & & & & & \\
\hline AI & -0.239 & -0.092 & $-\mathbf{- 0 . 1 0 3}$ & $\begin{array}{l}-018 \\
0.018\end{array}$ & 0.335 & $\overline{0} \mathbf{0 . 0 8 5}$ & 0.293 & -0.707 & $\mathbf{0 . 8 0 3}$ & 0.762 & 1 & & & & & & & \\
\hline $\mathbf{A M}$ & 0.199 & $\mathbf{0 . 0 8 7}$ & $\mathbf{0 . 3 5 3}$ & 0.541 & -0.624 & 0.812 & 0.086 & 0.529 & $\begin{array}{r}-0.840 \\
\end{array}$ & -0.004 & -0.650 & 1 & & & & & & \\
\hline CEC & 0.228 & 0.100 & -0.015 & $\begin{array}{l}- \\
0.254\end{array}$ & $\begin{array}{c}-0.139 \\
\end{array}$ & $\begin{array}{l}- \\
0.280\end{array}$ & -0.428 & 0.645 & -0.608 & -0.944 & -0.932 & 0.332 & 1 & & & & & \\
\hline $\mathbf{C A}$ & 0.930 & $0.971^{*}$ & 0.858 & $\begin{array}{l}- \\
0.790\end{array}$ & -0.675 & $\begin{array}{l}- \\
0.089\end{array}$ & \begin{tabular}{c|c|}
-0.888 \\
\end{tabular} & 0.616 & -0.296 & -0.002 & 0.095 & -0.148 & -0.021 & 1 & & & & \\
\hline MG & 0.875 & 0.865 & $0.984^{*}$ & $\begin{array}{l}\overline{0} \\
0.229\end{array}$ & $\overline{0.978 *}$ & 0.566 & -0.588 & 0.732 & -0.686 & 0.239 & -0.136 & 0.491 & -0.042 & 0.755 & 1 & & & \\
\hline NA & 0.897 & $0.951^{*}$ & 0.911 & $\overline{0.629}$ & -0.737 & 0.116 & -0.759 & 0.540 & -0.283 & 0.229 & 0.214 & -0.061 & -0.210 & $0.969^{*}$ & $\mathbf{0 . 8 3 9}$ & 1 & & \\
\hline $\mathbf{K}$ & 0.677 & 0.768 & 0.794 & $\begin{array}{l}-402 \\
0.402\end{array}$ & $\begin{array}{c}-0.599 \\
\end{array}$ & 0.252 & -0.466 & 0.225 & $-\mathbf{- 0 . 0 3 9}$ & 0.572 & 0.520 & $-\mathbf{- 0 . 1 3 3}$ & -0.561 & 0.820 & 0.751 & 0.926 & 1 & \\
\hline TNC & -0.884 & -0.923 & $\overline{0.966^{*}}$ & 0.438 & 0.848 & $\begin{array}{l}- \\
0.348\end{array}$ & 0.656 & -0.573 & 0.409 & $-\mathbf{- 0 . 3 3 3}$ & -0.155 & -0.152 & 0.240 & -0.897 & -0.934 & $\overline{0.971^{*}}$ & $\overline{-}$ & 1 \\
\hline
\end{tabular}

TFC - Total Fungal Colony, SA - Salinity, EC - Electrical Conductivity, OC - Organic Carbon, OM - Organic Matter, AN - Available Nitrogen, AP - Available Phosphorus, AK - Available Potassium, AZ - Available Zinc, AC - Available Copper, AI - Available Iron, AM - Available Manganese, CEC - Cat ion exchange capacity, CA - Calcium, MG - Magnesium, NA - Sodium, K - Potassium

** Correlation is significant at the 0.01 level. * Correlation is significant at the 0.05 level 
Int.J.Curr.Res.Aca.Rev.2016; 4(2): $x x-x x$

Table.10 Correlation of Physico - Chemical and Total Number of Colony Characteristics of the Soil Samples of Punnakayal in the Year of $2013-2014$

\begin{tabular}{|c|c|c|c|c|c|c|c|c|c|c|c|c|c|c|c|c|c|c|}
\hline & PH & SA & EC & OC & OM & AN & AP & AK & $\mathbf{A Z}$ & $\mathbf{A C}$ & AI & $\mathbf{A M}$ & CEC & CA & MG & NA & $\mathbf{K}$ & TNC \\
\hline PH & 1 & & & & & & & & & & & & & & & & & \\
\hline $\mathbf{S A}$ & 0.857 & 1 & & & & & & & & & & & & & & & & \\
\hline EC & 0.106 & 0.072 & 1 & & & & & & & & & & & & & & & \\
\hline OC & -0.922 & -0.917 & 0.209 & 1 & & & & & & & & & & & & & & \\
\hline OM & -0.871 & -0.908 & -0.456 & 0.767 & 1 & & & & & & & & & & & & & \\
\hline $\mathbf{A N}$ & -0.172 & -0.293 & -0.945 & -0.054 & 0.595 & 1 & & & & & & & & & & & & \\
\hline $\mathbf{A P}$ & 0.842 & 0.590 & -0.375 & -0.855 & $\begin{array}{l}\overline{0} .473 \\
\end{array}$ & 0.377 & 1 & & & & & & & & & & & \\
\hline AK & 0.922 & $0.989^{*}$ & 0.059 & $\mathrm{0.953}^{*}$ & $\begin{array}{l} \\
0.915\end{array}$ & -0.244 & 0.690 & 1 & & & & & & & & & & \\
\hline$\overline{\mathbf{A Z}}$ & $\begin{array}{l}-0.758 \\
\end{array}$ & -0.857 & 0.445 & $0.952^{*}$ & 0.592 & -0.241 & $\overline{0} \overline{0.780}$ & $\overline{0.867}$ & 1 & & & & & & & & & \\
\hline $\mathbf{A C}$ & 0.761 & 0.339 & -0.120 & -0.618 & $\begin{array}{l}- \\
0.387\end{array}$ & 0.253 & 0.903 & 0.473 & -0.448 & 1 & & & & & & & & \\
\hline $\mathbf{A I}$ & 0.454 & $\begin{array}{l}.119 \\
\end{array}$ & $\begin{array}{l}-0.649 \\
\end{array}$ & -0.505 & 0.042 & $\begin{array}{l}0.751 \\
\end{array}$ & $\begin{array}{l}0.859 \\
\end{array}$ & 0.234 & -0.504 & 0.826 & 1 & & & & & & & \\
\hline $\mathbf{A M}$ & -0.890 & -0.823 & 0.348 & $0.981^{*}$ & 0.646 & -0.230 & $\overline{0} .929$ & $\overline{0.877}$ & 0.949 & $\overline{0.704}$ & $\overline{0.659}$ & 1 & & & & & & \\
\hline CEC & 0.223 & 0.247 & $0.982^{*}$ & 0.058 & $\begin{array}{l}- \\
0.594\end{array}$ & $\overline{0.983}{ }^{*}$ & $\overline{-} .301$ & 0.223 & 0.285 & $\overline{0.112}$ & $\overline{0.653}$ & 0.216 & 1 & & & & & \\
\hline $\mathbf{C A}$ & 0.731 & 0.932 & -0.281 & -0.922 & $\begin{array}{l}- \\
0.695\end{array}$ & 0.027 & 0.626 & 0.911 & $\overline{0.966^{*}}$ & 0.273 & 0.266 & $\begin{array}{c}-0.870 \\
\end{array}$ & $\begin{array}{l}\overline{0} \\
0.101\end{array}$ & 1 & & & & \\
\hline MG & 0.836 & 0.729 & 0.632 & -0.613 & $\begin{array}{l}-943 \\
0.943\end{array}$ & $\begin{array}{l}-0.668 \\
\end{array}$ & 0.436 & 0.767 & -0.362 & 0.498 & $\begin{array}{l}- \\
0.029\end{array}$ & -0.505 & 0.720 & 0.441 & 1 & & & \\
\hline NA & $0.970^{*}$ & 0.798 & -0.130 & -0.948 & $\begin{array}{l}-735 \\
0.73\end{array}$ & 0.074 & 0.945 & 0.875 & -0.831 & 0.830 & 0.644 & $\overline{0.959 *}$ & $\overline{0}-\overline{0.019}$ & 0.751 & 0.680 & 1 & & \\
\hline $\mathbf{K}$ & 0.040 & 0.371 & 0.731 & 0.028 & $\begin{array}{l}- \\
0.526\end{array}$ & $\begin{array}{c}-0.905 \\
\end{array}$ & $\begin{array}{l} \\
0.492\end{array}$ & 0.270 & 0.095 & $\begin{array}{l}- \\
0.537\end{array}$ & $\begin{array}{l}- \\
0.869\end{array}$ & 0.220 & 0.811 & 0.160 & 0.464 & $\begin{array}{l} \\
0.181\end{array}$ & 1 & \\
\hline TNC & -0.856 & -0.531 & $\begin{array}{c}-0.440 \\
\end{array}$ & 0.591 & 0.749 & 0.353 & $\overline{0}-660$ & $\overline{0.629}$ & 0.318 & $\begin{array}{l}- \\
0.815\end{array}$ & $\begin{array}{l} \\
0.351\end{array}$ & 0.566 & $\overline{0.475}$ & $\overline{0.279}$ & $\overline{0}-\overline{0.890}$ & $\begin{array}{l} \\
0.775\end{array}$ & $\overline{0} \overline{0.032}$ & 1 \\
\hline
\end{tabular}

TFC - Total Fungal Colony, SA - Salinity, EC - Electrical Conductivity, OC - Organic Carbon, OM - Organic Matter, AN - Available Nitrogen, AP - Available Phosphorus, AK - Available Potassium, AZ - Available Zinc, AC - Available Copper, AI - Available Iron, AM - Available Manganese, CEC - Cat ion exchange capacity, CA - Calcium, MG - Magnesium, NA - Sodium, K Potassium

** Correlation is significant at the 0.01 level. * Correlation is significant at the 0.05 level 
Int.J.Curr.Res.Aca.Rev.2016; 4(2): $x x-x x$

Table.11 Correlation of Physico - Chemical and Total Number of Colony Characteristics of the Soil Samples of Tuticorin in the Year of $2013-2014$

\begin{tabular}{|c|c|c|c|c|c|c|c|c|c|c|c|c|c|c|c|c|c|c|}
\hline & PH & SA & EC & OC & OM & $\mathbf{A N}$ & $\mathbf{A P}$ & AK & $\mathbf{A Z}$ & AC & AI & $\mathbf{A M}$ & CEC & CA & MG & NA & $\mathbf{K}$ & TNC \\
\hline PH & 1 & & & & & & & & & & & & & & & & & \\
\hline SA & 0.740 & 1 & & & & & & & & & & & & & & & & \\
\hline EC & 0.594 & 0.368 & 1 & & & & & & & & & & & & & & & \\
\hline OC & $\begin{array}{l}-0.869 \\
\end{array}$ & -0.506 & -0.179 & 1 & & & & & & & & & & & & & & \\
\hline OM & $\overline{0.989 *}$ & -0.634 & -0.574 & 0.905 & 1 & & & & & & & & & & & & & \\
\hline $\mathbf{A N}$ & -0.110 & 0.397 & -0.694 & $\begin{array}{l}- \\
0.071\end{array}$ & 0.188 & 1 & & & & & & & & & & & & \\
\hline AP & 0.546 & -0.024 & 0.826 & $\overline{0.360}$ & -0.613 & -0.891 & 1 & & & & & & & & & & & \\
\hline AK & $0.965^{*}$ & 0.889 & 0.569 & $\overline{0} \mathbf{0 . 7 7 2}$ & -0.915 & 0.058 & 0.377 & 1 & & & & & & & & & & \\
\hline $\mathbf{A Z}$ & 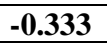 & $\begin{array}{l}-0.307 \\
\end{array}$ & 0.561 & 0.693 & 0.349 & $\begin{array}{l}-0.679 \\
\end{array}$ & 0.387 & -0.316 & 1 & & & & & & & & & \\
\hline AC & 0.261 & -0.452 & 0.173 & $\begin{array}{l}\overline{0} \\
0.470\end{array}$ & -0.401 & -0.639 & 0.697 & -0.001 & $\begin{array}{l}\overline{0} \\
\mathbf{0 . 0 9 8}\end{array}$ & 1 & & & & & & & & \\
\hline AI & 0.661 & $0.967^{*}$ & 0.512 & $\overline{0} \mathbf{0 . 3 2 1}$ & -0.543 & 0.263 & 0.042 & 0.830 & $\begin{array}{l} \\
0.056\end{array}$ & -0.534 & 1 & & & & & & & \\
\hline $\mathbf{A M}$ & 0.082 & 0.477 & -0.640 & $\overline{0.297}$ & -0.019 & $\mathbf{0 . 9 7 3}^{*}$ & $\overline{0.775}$ & 0.217 & $\overline{0.817}$ & -0.491 & 0.303 & 1 & & & & & & \\
\hline CEC & -0.670 & -0.433 & 0.179 & 0.933 & 0.702 & $\begin{array}{c}-0.370 \\
\end{array}$ & $\begin{array}{l}- \\
0.025\end{array}$ & -0.600 & 0.905 & $\begin{array}{c}-0.342 \\
\end{array}$ & -0.197 & -0.572 & 1 & & & & & \\
\hline CA & 0.705 & 0.513 & $0.985^{*}$ & $\begin{array}{l}-297 \\
\end{array}$ & -0.674 & -0.579 & 0.779 & 0.699 & 0.432 & 0.122 & 0.630 & -0.504 & 0.048 & 1 & & & & \\
\hline MG & 0.199 & -0.171 & 0.853 & 0.116 & -0.236 & $\overline{0.955^{*}}$ & 0.878 & 0.095 & 0.781 & 0.418 & 0.004 & -0.947 & 0.453 & 0.754 & 1 & & & \\
\hline NA & $0.954^{*}$ & 0.514 & 0.663 & $\begin{array}{l}-851 \\
0.051\end{array}$ & $\overline{0.982^{*}}$ & -0.367 & 0.749 & 0.849 & $\begin{array}{l} \\
0.214\end{array}$ & 0.515 & 0.448 & -0.164 & -0.606 & 0.736 & 0.398 & 1 & & \\
\hline $\mathbf{K}$ & 0.808 & 0.736 & 0.898 & $\begin{array}{l}- \\
0.412\end{array}$ & -0.749 & -0.326 & 0.610 & 0.850 & 0.231 & -0.045 & 0.815 & -0.238 & -0.116 & $\mathbf{0 . 9 5 8}^{*}$ & 0.537 & 0.758 & 1 & \\
\hline TNC & -0.893 & -0.901 & -0.712 & 0.579 & 0.821 & 0.035 & $\overline{0.410}$ & $\overline{0.963}{ }^{*}$ & 0.071 & 0.138 & -0.911 & $\begin{array}{c}-0.079 \\
\end{array}$ & 0.362 & $\begin{array}{l}-0.820 \\
\end{array}$ & -0.245 & -0.773 & -0.947 & 1 \\
\hline
\end{tabular}

TFC - Total Fungal Colony, SA - Salinity, EC - Electrical Conductivity, OC - Organic Carbon, OM - Organic Matter, AN - Available Nitrogen, AP - Available Phosphorus, AK - Available Potassium, AZ - Available Zinc, AC - Available Copper, AI - Available Iron, AM - Available Manganese, CEC - Cat ion exchange capacity, CA - Calcium, MG - Magnesium, NA - Sodium, K Potassium

** Correlation is significant at the 0.01 level. * Correlation is significant at the 0.05 level. 
Int.J.Curr.Res.Aca.Rev.2016; 4(2): $x x-x x$

The results of the present study demonstrated that the genus Aspergillus (17 species) and Penicillium (7 species) were dominantly isolated. This is in agreement with the previous finding of Thennarasu et al. (2011) and Anitha and Nayak (2014) who reported that Aspergillus and Penicillium were dominant genera in coastal area of Andaman Islands and Puducherry and Karaikal region.

Percentage frequency and frequency class of different species of fungi were evaluated (Table 5). A. niger was commonly isolated from all the three stations. The $\mathrm{pH}$ of soil was ranged from 8.22 to 8.72. The major and minor elements were showed variations in the study stations (Table 6-8). The relationship between various physicochemical parameters of soil and total fungal colonies were statistically analyzed (9-11).

Many factors can influence the activity, abundance and distribution of fungi in the marine environment. Salinity and temperature are the major factors affecting the diversity of marine fungi as is well illustrated by the data of Booth and Kenkel (1986). In the present study, Sodium ( $\mathrm{r}=-$ $0.971 ; \mathrm{P}<0.05)$, and electrical conductivity $(\mathrm{r}=-0.966 ; \quad \mathrm{P}<0.05) \quad$ showed negative correlation at Palayakayal station and available potassium $(\mathrm{r}=-0.963 ; \mathrm{P}<0.05)$ exhibited negative correlation at Tuticorin station. From the present study, it appears that the coastal soils contain a mycoflora reservoir comprising of a variety of genera. Tuticorin coast provides a unique opportunity for mycologist to explore fungal diversity and exploit their ecological, medicinal and industrial potential.

\section{References}

Anitha, K. and Nayak, B. K., 2014. Diversity of sand dune fungi in the coastal areas of Puducherry and Karaikal region. Advances in Applied Science Research, 5(5):170-176.

APHA, 1989. Standard Methods for the Examination of Water and Wastewater. $17^{\text {th }}$ edition, American Public Health Association, USA.

Ashok, G., Senthilkumar, G. and Panneerselvam, A.,2015. Diversity and Seasonal Variation of Soil Fungi Isolated from Coastal Area of Tuticorin Dt., Tamil Nadu, India. Int. J. Curr. Microbiol. App. Sci., 4(10): 161-178.

Bass, D., Howie, A., Brown, N., Barton, N. and Demidoba, M., 2007. Yeast forms dominate fungal diversity in the deep oceans. Proceedings of the Royal Society B. 274:3069-3077.

Behera, B. C., Mishra, R. R. and Thatoi, H. N., 2012. Diversity of soil fungi from mangroves of Mahanadi delta, Orissa. India J. Microbiol. Biotech. Res., 2 (3):375-378.

Booth, T. and Kenkel, N., 1986. Ecological studies of lignicolous marine fungi: a distribution model based on ordination and classification. In: The Biology of Marine Fungi (ed. S.T. Moss). Cambridge University Press, Cambridge: 297-310.

Borse, B.D. and N.S. Pawar, 2001. Carbosphaerella and DryosphaeraTwo New Generic records of marine ascomycetes from West Bengal (India), Geiobios, 28: 117-120.

Borse, B.D., D.J. Kelkar and A.C. Patil, 2000. Frequency of occurrence of marine fungi from Pirotan island (Gujarat), India, Geiobios, 27: 145148.

Borse, B.D., S.N. Nandan and D.N. Shinde, 1999. Higher marine fungi from Goa coast (India) BRI's JAST 2: 52-55.

Domsch, K.H., Gams, W. and Anderson, T.H., 1980. Compendium of soil 
Int.J.Curr.Res.Aca.Rev.2016; 4(2): $x x-x x$

fungi. Academic Press, New York, USA.

Ellis, M.B, 1976. More Dematiaceous Hyphopmycetes. Commonwealth Mycological Institute, Kew, Surrey, England.

Ellis, M.B., 1971. Dematiaceous hyphomycetes. Commonwealth Mycological Institute, Kew, Surrey, England.

Fatma, F. M., 2003. Distribution of fungi in the sandy soil in Egyptian beaches. Pakistan Journal of Biological Sciences, 6(10):860-866.

Gillman, J.C., 1957. A Manual of Soil Fungi. Revised $2^{\text {nd }}$ edition Oxford and IBH publishing company (Indian reprint) Calcutta, Bombay, New Delhi.

Gomes, D. N. F., Cavalcanti, M. A. Q., Fernandes, M. J. S., Lima, D. M. M. and Passavante, J. Z. O., 2008. Filamentous fungi isolated from sand and water of Bairro Novo and Casa Caiada beaches, Olinda, Pernambuco, Brazil. Brazlian Journal of Biology. 68(3):577-582.

Hyde, K. D., Jones, E. B. G., Leaňo, E., Pointing, S. B. and Poonyth, A. D., 1998. Role of fungi in marine ecosystems. Biodiversity and Conservation. 7:1147-1161.

Hyde, K.D., Sharma, V. V., Jones, E. B. G. and Hyde, K. D., Pointing, S. B. 2000. Morphology and taxonomy of higher marine fungi. Marine mycology: a practical approach: Fungal Diversity Press..

Jones E. B. G. and Pang K.L., 2012. Marine Fungi: and Fungal-like Organisms (Marine and Freshwater Botany). Berlin: Walter de Gruyter, 528.

Jones, E. B. G., 2011. Fifty years of marine mycology. Fungal Divers., 50:73112.
Jones, E.B.G., Sakayaroj, J., Suetrong, S., Somrithipol, S. and Pang, K. L., 2009. Classification of marine ascomycota, anamorphic taxa and basidiomycota. Fungal Divers., 35:1187.

Kohlmeyer, J. and Kohlymeyer, E., 1979. Marine Mycology, The higher fungi. Academic Press, New York, U.S.A.

Larrondo, J. V. and Calvo, M. A., 1989. Fungal density in the sands of the Mediterranean coast beaches. Mycopathologia, 108(3):185-193.

Mancini, L., D’Angelo, A.M., Pierdominici, E., Ferrari, C., Anselmo, A., Venturi, L., Fazzo, L., Formichetti, P., Iaconelli, M. and Panelli, B., 2005. Microbiological quality of Italian beach sands. Microchemical Journal., 79(1-2):259-261.

Menezes, C. B. A., Bonugli-Santos, R. C., Miqueletto, P. B., Passarini, M. R. Z., Silva, C. H. D. and Justo, M. R., 2010. Microbial diversity associated with algae, ascidians and sponges from the north coast of Sao Paulo state, Brazil. Microbiol. Res., 165: 466-482.

Morrison-Gardiner, S., 2002. Dominant fungi from Australian coral reefs. Fungal Divers. 9: 105-121.

Papadakis, J. A., Mavridou, A., Richardson, S. C., Lambiri, M. and Marcelou U., 1997. Bather-related microbial and yeast populations in sand and seawater. Water Research. 31(4):799-804.

Patil, S.D. and B.D. Borse, 1983. Marine fungi from Maharashtra (India)-II. Some arenicolous fungi, Indian bot. Rept., 2: 83-87.

Prasannarai, K. and K.R. Sridhar, 1993. Marine fungi from decaying wood from Mangalore coast. Mahasagar, 24: 33-38. 
Int.J.Curr.Res.Aca.Rev.2016; 4(2): $x x-x x$

Raper, K.B. and Fennell, D.I., 1965. The genus Aspergillus, The Williams and Wilkins Co., Baltimore, U.S.A.

Raper, K.B. and Thom, C., 1949. A Manual of Penicillia. Williams and Wilkins Co., Baltimore, Md., U.S.A.

Raveendran, K. and P. Manimohan, 2007. Marine fungi of Kerala-A preliminary floristic and ecological study (Malabar Natural History Society, Calicut, Kerala).

Ravikumar, D.R. and B.P.R. Vittal, 1996. Fungal diversity on decomposing biomass of mangrove plant Rhizophora in Pichavaram estuary, east coast of India. Indian J. Mar. Sci., 25: 142-146.

Richards T. A., Jones M. D. M., Leonard G. and Bass D., 2012. Marine fungi: their ecology and molecular diversity. Ann. Rev. Mar. Sci., 4: 495-522.

Sarma, V.V. and B.P.R. Vittal, 2001. Biodiversity of manglicolous fungi on selected plants in the Godavari and Krishna delta east coast of India, Fungal Diversity, 6: 115-130.

Subramanian, C.V., 1971. Hypomycetes: An account of Indian species, Indian Counc. Agri. Res., New Delhi.
Tedersoo, L., Anslan, S., Bahram, M., Põlme, S. and Riit, T., 2015. Shotgun metagenomes and multiple primer pair-barcode combinations of amplicons reveal biases in metabarcoding analyses of fungi. MycoKeys, (10):1-43.

Tedersoo, L., Bahram, M., Polme, S., Anslan, S. and Riit, T., 2014. Global diversity and geography of soil fungi. Science, 936.

Thennarasu, V., Panneerselvam, A. and Thajuddin, N., 2011. An investigation of the mycoflora in marine soil from Andaman Islands. Euro. J. Exp. Bio., 1(3): 188199.

Warcup, J.H., 1950. The soil plate method for isolation of fungi from soil. Nature, 166: 117-117.

Zakaria, L., Yee, T. L., Zakaria, M. and Salleh, B., 2011. Diversity of Microfungi in Sandy Beach Soil of Teluk Aling, Pulau Pinang. Tropical Life Sciences Research, 22(1), 71-80.

\section{How to cite this article:}

Ashok, G., G. Senthilkumar, A. Panneerselvam and Umamagheswari, K. 2016. Studies on Soil Mycoflora in Coastal Area of Tuticorin Dt., Tamilnadu, India. Int.J.Curr.Res.Aca.Rev.4(2): 3955. doi: http://dx.doi.org/10.20546/ijcrar.2016.402.005 